\title{
Potencial de Formigas (Hymenoptera: Formicidae) como Agentes Anti-herbívoros em Cultivo de Café (Coffea canephora Pierre) e Feijão Guandu [Cajanus cajans (L.) Millsp]
}

\author{
Leandro Xavier Teixeira Chevalier, Diego Silva Gomes, Antonio José Mayhé-Nunes \& Jarbas Marçal Queiroz ${ }^{\bowtie}$
}

1. Universidade Federal Rural do Rio de Janeiro, e-mail: leandrochevalierufrrj@yahoo.com.br, dyegouss@yahoo.com.br, amaynun@gmail.com, jarquiz@gmail.com (Autor para correspondência ${ }^{\varpi}$ ).

\section{EntomoBrasilis 6(2): 113-118 (2013)}

Resumo. Este trabalho analisou a variação no potencial das formigas como agentes anti-herbívoros em plantas de café (Coffea canephora Pierre) e guandu [Cajanus cajans (L.) Millsp]. Foram usados ovos artificiais, simulando a presença de insetos herbívoros, e solução de açúcar, para aumentar a atividade de formigas, em experimentos de campo. As plantas de café e de guandu possuíam diferentes níveis de atividade de formigas, pois as últimas eram atacadas por membracídeos que atraíam mais formigas. A atividade das formigas foi monitorada após aplicação de solução de açúcar e a remoção de ovos artificiais foi medida após 24 e 48 horas. A solução de açúcar aumentou significativamente a atividade de formigas em plantas de café e de guandu. A remoção dos ovos artificiais foi significativamente maior em ramos com formigas do que sem formigas para as plantas de guandu. A adição de solução de açúcar aumentou a remoção de ovos artificiais em plantas de guandu, mas não em plantas de café. O tratamento com solução de açúcar e a presença de membracídeos em guandu tiveram efeitos similares sobre a atividade de formigas. Este estudo mostrou que a aplicação de solução de açúcar pode ser utilizada para simular a presença de membracídeos sobre as plantas, aumentando a atividade de formigas. Isso permitiu a avaliação de seu potencial como agentes anti-herbívoros, através de experimentos que simulam a presença de insetos sobre as plantas.

Palavras-Chave: Controle biológico; Exclusão de predadores; Interação inseto-planta.

\section{Potential of Ants (Hymenoptera: Formicidae) as Anti-herbivore Agents in Cultivation of the Coffee (Coffea canephora Pierre) and Pigeon Pea [Cajanus cajans (L.) Millsp]}

Abstract. This study analyzed the role of ants as anti-herbivore agents in Coffee (Coffea canephora Pierre) and Pigeon Pea [Cajanus cajans (L.) Millsp] plants in agroecosystem under organic management. In these plants we used artificial eggs and sugar solution in experiments that simulated the presence of herbivore insects. Coffee plants did not possess any natural attractive that could intensify foraging activity of ants, but pigeon pea plants were infested by treehoppers which attracted ants. Activity of ants was surveyed after sugar solution application and artificial eggs removal was measured 24 and $48 \mathrm{~h}$ after start of the experiments. Sugar solution sprinkled on plants foliage increased significantly the activity of ants on coffee and pigeon pea plants. The removal of artificial eggs was significantly greater in branch with ants than without ants for pigeon pea plants. The sugar solution addiction resulted in greater artificial eggs removal in pigeon pea plants, but not in coffee plants. The treatment with sugar solution and presence of treehoppers had similar effects on the activity of ants on the plants. This study showed that the application of sugar solution can be used to increase activity of ants on plants as well as the potential of ants as anti-herbivore agents can be evaluated in experiments that simulate the presence of insects on the plants.

Keywords: Biological control; Insect-plant interaction; Predator exclusion.

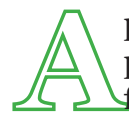

presença de formigas forrageando sobre as folhagens de plantas está frequentemente associada à existência de fontes renováveis de alimento, como néctar e exsudados de insetos herbívoros - "honeydew" (BENTLEY 1977; Buckley 1987; Oliveira \& Freitas 2004; Dutra et al. 2006). Em outros casos, as plantas podem prover abrigos em estruturas nas quais as formigas nidificam (HöLldobler \& WiLson 1990). Evidências do papel benéfico de formigas para plantas já foram descritas tanto para ecossistemas nativos (Koptur 1984), quanto para agroecossistemas (IBARRA-NúNEZ et al. 2001), pois as formigas podem predar insetos herbívoros e reduzir os danos causados às plantas. Devido a esse potencial em reduzir o tamanho das populações de insetos herbívoros, as formigas são consideradas importantes agentes de controle biológico (VANDERMEER et al. 2002; PhILPOTT \& ARMBRECHT 2006).

Em agroecossistemas, as atividades agrícolas contribuem para uma redução na diversidade de formigas (QueIroz et al. 2006) podendo afetar negativamente o potencial da mirmecofauna em causar mortalidadenas populações deinsetos herbívoros (РніцРотт et al. 2006; Philpott \& Armbrecht 2006). No entanto, dados empíricos sobre os condicionantes para variações no impacto das formigas como predadores de insetos em agroecossistemas ainda são escassos. Neste trabalho o objetivo foi avaliar o potencial das formigas como agentes anti-herbívoros em cultivos de café (Coffea canephora Pierre) e feijão guandu [Cajanus cajans (L.) Millsp].

\section{MATERIAL E MÉTODOS}

Área de estudo e organismos. O estudo foi realizado entre os meses de Março de 2007 a Junho de 2008 no Sistema Integrado de Produção Agroecológica (SIPA), localizado em Seropédica, RJ, $\left(22^{\circ} 46^{\prime} \mathrm{S}\right.$, $43^{\circ} 41^{\prime} \mathrm{O} ; 33 \mathrm{~m}$ de altitude) (REsende et al. 2006). Foram utilizadas $43^{\circ} 41^{\prime} \mathrm{O} ; 33 \mathrm{~m}$ de altitude) (RESENDE et al. 2006). Foram utilizadas

Agência(s) de Financiamento: FAPERJ - bolsa de Iniciação Cientifica Proc. 151.902/2006 e DSG (Proc. 150.902/2007) e CNPq. 

- cultivo sazonal, estando elas distantes 200 metros uma da outra. As plantas de café não possuíam nenhum atraente natural para formigas como, por exemplo, flores, nectários extraflorais ou exsudatos de insetos. No caso do feijão guandu, parte das plantas encontrava-se com duas espécies de membracídeos que atraíam formigas: Enchenopa gracilis (Germar) e Calloconophora argentipennis Dietrich. As duas espécies foram identificadas pelo Dr. Albino Sakakibara e depositadas na Coleção Entomológica Pe. Jesus Santiago Moure (UFPR).

Os exemplares de cada morfoespécie de formiga encontrados sobre as plantas experimentais foram montados em via seca. As identificações foram realizadas ao nível de gênero com base na chave de Bolton (1994), LAPOlla et al. (2010) e, quando possível, ao nível de espécie por meio de comparações com exemplares identificados da Coleção Entomológica Costa Lima (CECL)/ UFRRJ e com o auxílio do Dr. Jacques Delabie (CEPEC/CEPLAC). O material testemunho foi depositado na Coleção Entomológica Costa Lima (CECL)/UFRRJ.

Procedimentos experimentais. Foi aplicada uma solução de açúcar sobre as plantas, visando aumentar artificialmente a atividade de formigas sobre as plantas. Para isso, foram utilizados 20 pares de plantas de café e 20 pares de plantas de feijão guandu (sendo 12 pares desta cultura com membracídeos e oito sem membracídeos), cada par com uma distância média de $10 \mathrm{~m}$ um do outro. As plantas foram inspecionadas durante o experimento para garantir que não houvesse colonização por membracídeos das plantas inicialmente sem membracídeos. Foi borrifada solução de açúcar, preparada com concentração de 1:10 (10 g de açúcar em $100 \mathrm{~mL}$ de água sem tratamento de cloro), por toda a extensão de uma das plantas de cada par, até que todas as folhas estivessem úmidas, na outra planta foi borrifada somente água. A atividade das formigas foi avaliada pelo número de operárias que transitavam pela planta durante 24 minutos. A duração do tempo de observação foi determinada baseada em trabalho anterior (Oliveira et al. 1987) e em estudos preliminares de medição do tempo necessário para os deslocamentos entre os grupos experimentais.

Foram confeccionados manualmente, com massa de modelar branca, ovos artificiais, de formato arredondado, com diâmetro médio de $3 \mathrm{~mm}$, a fim de simular ovos de insetos herbívoros, para testar a técnica. $\mathrm{O}$ tamanho dos ovos artificiais foi baseado no tamanho dos ovos de Isognathus caricae L. (Lepidoptera: Sphingidae). Massas de modelar já foram utilizadas em experimentos ecológicos para avaliação de predação (ANDRADE 1997; GALETTI et al. 2003). Estes ovos foram fixados nos 20 pares de plantas de cada cultura, os mesmos pares usados no experimento da atividade de formigas. Em cada planta foram selecionados dois ramos, em um deles foi aplicada a resina Tanglefoot ${ }^{\circledR}$, que impede que formigas acessem o ramo (exclusão de formigas). Em cada ramo foram fixados, com cola plástica, três ovos artificiais em folhas diferentes. Ramos ou folhas próximas que pudessem dar acesso às formigas para os ramos isolados foram removidos. O número de ovos artificiais removidos foi contabilizado após 24 horas e, com reposição dos ovos removidos, após 48 horas do início do experimento.

Análises Estatísticas. Os dados não apresentaram distribuição normal, por isso, foram analisados pelo teste não-paramétrico de Mann Whitney, a 5\% de probabilidade. O número de ocorrência das diferentes espécies de formigas nas plantas de C. canephora e C. cajans foi contabilizado como o número de plantas em que cada espécie foi observada. O número de operárias que transitavam pelas plantas foi utilizado para comparação da atividade entre os tratamentos (solução de açúcar e água) e entre as culturas (café e feijão guandu); e o número de ovos artificiais removidos foi utilizado nas análises para comparação entre os ramos isolados e não isolados de formigas nas plantas. Todas as análises foram realizadas com o uso do programa SYSTAT ${ }^{\circledR}$ (versão 8.o).

\section{RESULTADOS}

Foram encontradas 17 espécies de formigas, sendo cinco sobre plantas de café e 16 sobre plantas de feijão guandu. Nas plantas de café, Monomorium sp. e Camponotus crassus Mayr foram as espécies mais frequentes. Nas plantas de feijão guandu as espécies mais frequentes foram Brachymyrmex sp.1, C. crassus e C. rufipes (Tabela 1).

Tabela 1. Formigas encontradas sobre as plantas experimentais de Café (Coffea canephora Pierre) e Feijão Guandu [Cajanus cajans (L.) Millsp] em Seropédica, RJ. Março de 2007 a Junho de 2008.

\begin{tabular}{lcc}
\hline \multicolumn{1}{c}{ Espécies de formigas } & Café & $\begin{array}{c}\text { Feijão } \\
\text { guandu }\end{array}$ \\
\hline Brachymyrmex sp.1 & $\mathrm{X}$ & $\mathrm{X}$ \\
\hline Brachymyrmex sp. 2 & $\mathrm{X}$ & $\mathrm{X}$ \\
\hline Camponotus crassus Mayr & $\mathrm{X}$ & $\mathrm{X}$ \\
\hline Camponotus melanoticus Emery & & $\mathrm{X}$ \\
\hline Camponotus rufipes (Fabricius) & & $\mathrm{X}$ \\
\hline Camponotus sp. & & $\mathrm{X}$ \\
\hline Cardiocondyla sp. & $\mathrm{X}$ & $\mathrm{X}$ \\
\hline Crematogaster sp. & $\mathrm{X}$ & $\mathrm{X}$ \\
\hline Monomorium sp. & & $\mathrm{X}$ \\
\hline Paratrechina sp. & & $\mathrm{X}$ \\
\hline Pheidole sp.1 & & $\mathrm{X}$ \\
\hline Pheidole sp.2 & & $\mathrm{X}$ \\
\hline Pheidole sp.3 & & $\mathrm{X}$ \\
\hline Pheidole sp.4 & & $\mathrm{X}$ \\
\hline Pseudomyrmex sp.1 & & $\mathrm{X}$ \\
\hline Solenopsis sp. & & $\mathrm{X}$ \\
\hline
\end{tabular}

Efeitos da solução de açúcar na atividade de formigas sobre plantas. As culturas diferiram estatisticamente em relação à atividade de formigas, havendo 2,5 vezes mais formigas nas plantas de feijão guandu, com e sem membracídeos, do que nas plantas de café (Teste Mann Whitney, $\mathrm{P}=0$,015). A média da atividade de formigas nas plantas de café e de feijão guandu foi significativamente maior em plantas borrifadas com solução de açúcar (Teste Mann Whitney - Café, $\mathrm{P}=0$,012; Feijão guandu, $\mathrm{P}$ $=0,018$; Figura 1 ).

Nas plantas de feijão guandu, a atividade de formigas também foi maior em plantas com membracídeos do que sem membracídeos (Teste Mann Whitney, $\mathrm{P}=0,017$ ). Não houve diferença significativa entre a atividade de formigas em plantas com membracídeos (sem solução de açúcar) ou apenas com solução de açúcar (sem membracídeos) (Teste Mann Whitney, P = 0,149).

Teste de ovos artificiais para medir o potencial das formigas como agentes anti-herbívoros. Na comparação entre ramos isolados pela resina Tanglefoot $\AA$ (com exclusão de formigas) e não isolados (sem exclusão), a média do número de ovos artificiais removidos foi significativamente maior, após 48 horas, nos ramos não isolados nas plantas de feijão guandu (Teste Mann Whitney, $\mathrm{P}=0,008$ ), mas não houve diferença para as plantas de café (Teste Mann Whitney, $\mathrm{P}=0,100$ ) (Figura 2).

Além disso, o tratamento com solução de açúcar resultou em um número médio de ovos artificiais removidos maior do que no tratamento com água em plantas de feijão guandu (Teste Mann Whitney, P = 0,005), mas novamente não houve diferença significativa para as plantas de café (Teste Mann Whitney, $\mathrm{P}=$ 


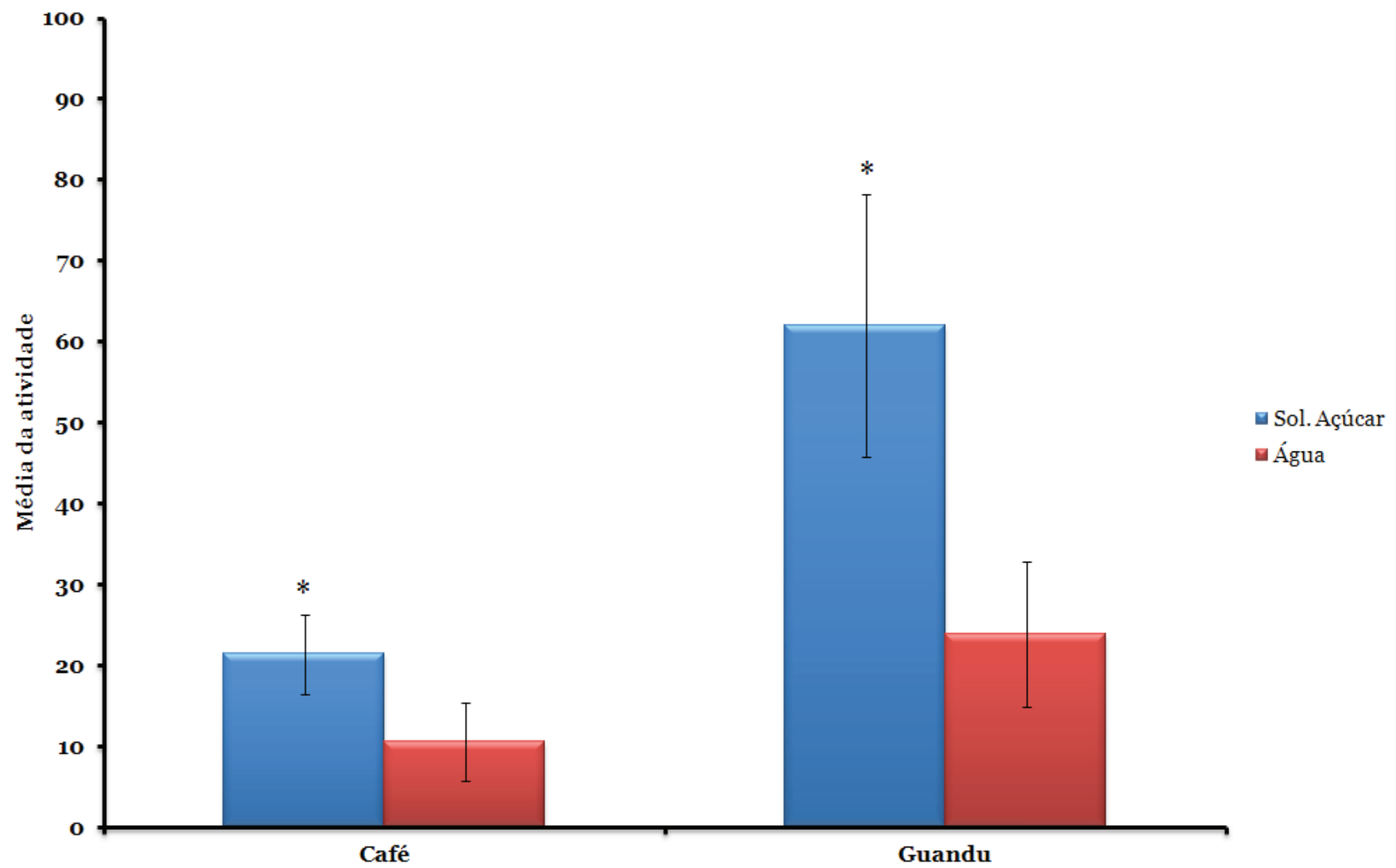

Figura 1. Valor médio ( \pm erro padrão) da atividade de formigas em plantas de Café (Coffea canephora Pierre) e Feijão Guandu [Cajanus cajans (L.) Millsp] em Seropédica, RJ. Março de 2007 a Junho de 2008. *representa diferença estatística (P < 0,05).

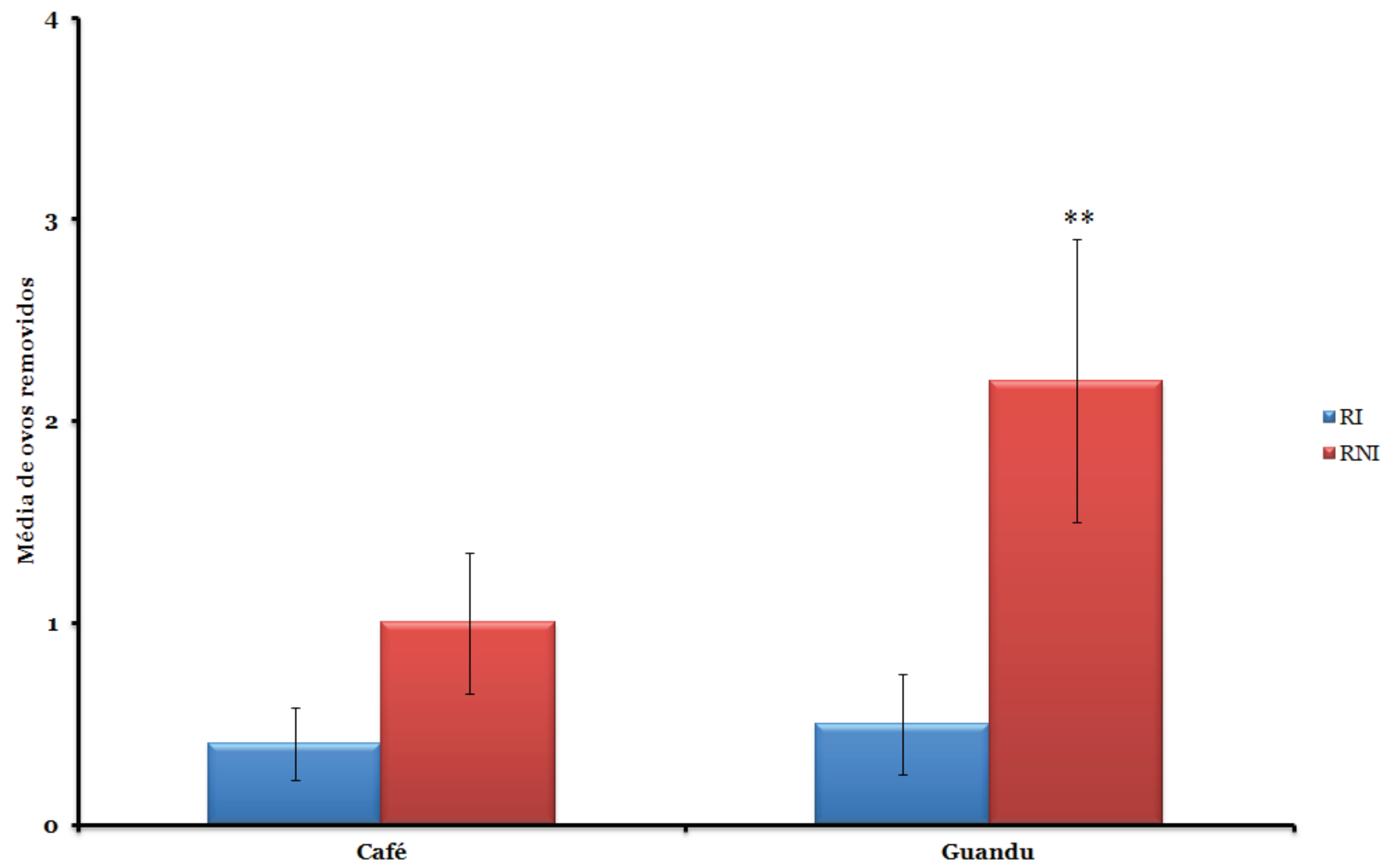

Figura 2. Número médio ( \pm erro padrão) de ovos artificiais removidos dos ramos isolados (RI) e não isolados (RNI) de formigas, nas plantas de Café (Coffea canephora Pierre) e Feijão Guandu [Cajanus cajans (L.) Millsp] em Seropédica, RJ. Março de 2007 a Junho de 2008. ${ }^{* *}$ representa diferença estatística $(\mathrm{P}<0,01)$ 
Quando analisada apenas a remoção nos ramos não isolados

nas plantas de feijão guandu com ou sem membracídeos, houve diferença significativa no número médio de ovos removidos entre os tratamentos (solução de açúcar e água) apenas para as

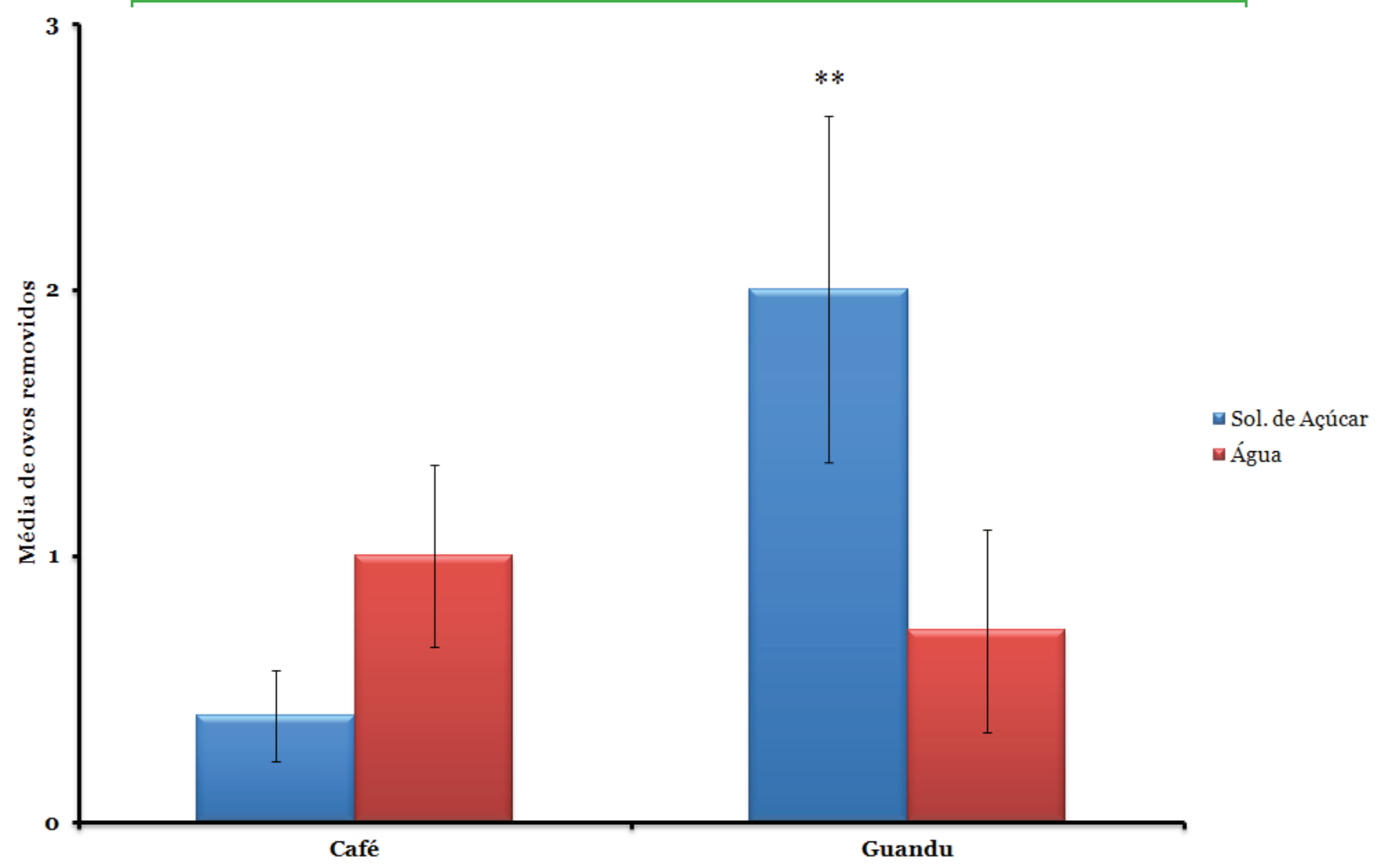

Figura 3. Número médio ( \pm erro padrão) de ovos artificiais removidos dos ramos nas plantas de nas plantas de Café (Coffea canephora Pierre) e Feijão Guandu [Cajanus cajans (L.) Millsp] em Seropédica, RJ, cujas folhas foram tratadas com solução de açúcar ou água. Março de 2007 a Junho de 2008. ** representa diferença estatística $(\mathrm{P}<0,01)$.

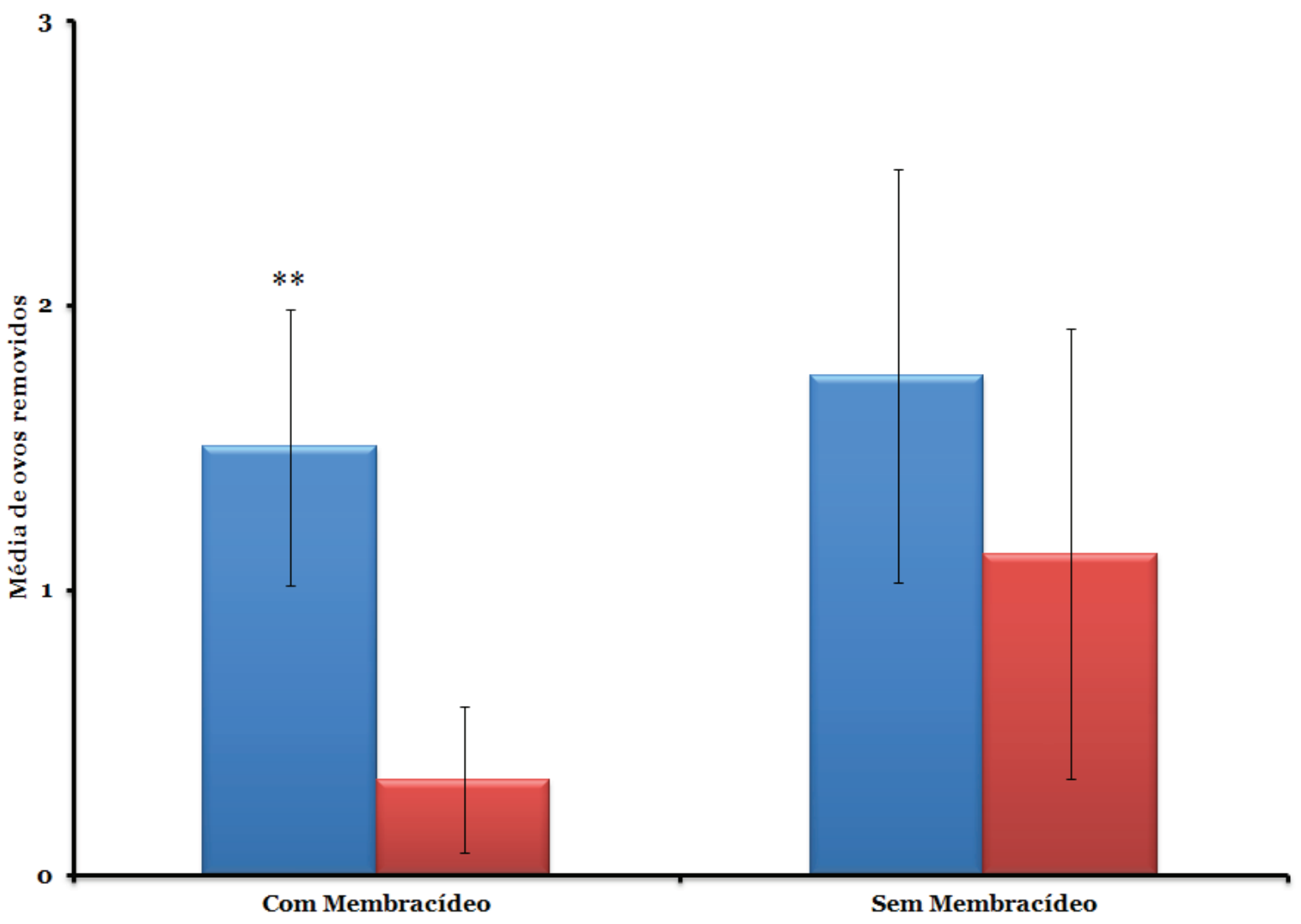

অ Sol. Açúcar घÁgua

Figura 4. Número médio ( \pm erro padrão) de ovos removidos nas plantas de Feijão Guandu [Cajanus cajans (L.) Millsp] em Seropédica, RJ, cujas folhas foram tratadas com solução de açúcar ou água. Março de 2007 a Junho de $2008 .{ }^{* *}$ representa diferença estatística $(\mathrm{P}<\mathrm{O}, 01)$. 
plantas com presença de membracídeos (Teste Mann Whitney, $\mathrm{P}$ $=0,009$; Figura 4).

\section{DISCUSSÃO}

Estudos experimentais sobre predação na naturezajá se utilizaram de modelos de massa plástica para avaliações rápidas (ANDRADE 1997; GALETTI et al. 2003). No caso de estudos envolvendo a função de formigas como predadores, iscas de cupins vivos em ecossistemas nativos (OliveIRA et al. 1987; JunQueIRA et al. 2001) ou lagartas polífagas em agroecossistemas (VANDERMEER et al. 2002) já haviam sido utilizados, mas não modelos de massa plástica como os empregados neste trabalho. Esses modelos simularam a presença de ovos de insetos herbívoros que poderiam ou não ser removidos por formigas. Estudos que utilizaram cupins vivos para medir o potencial das formigas como agentes antiherbívoros sobre plantas mostraram resposta quase que imediata das formigas à introdução destes organismos sobre as plantas (e.g. Costa et al. 1992; MACHADO \& FREITAS 2001). No experimento realizado neste trabalho, as diferenças encontradas no número de ovos desaparecidos em ramos isolados e não isolados de formigas demonstraram a função que as formigas podem exercer como agentes anti-herbívoros em um período de 48 horas. No entanto, como demonstrado em outros estudos (e.g. RASHBROOK et al. 1992; QueIroz \& OliveIra 2001), os efeitos da formigas sobre potenciais herbívoros são amplamente condicionados pelo nível de atividade dessas sobre as plantas.

Em trabalho anterior, os autores manipularam o nível de atividade de formigas sobre plantas utilizando pedaços de sardinha (VANDERMEER et al. 2002). Neste trabalho, conseguiu-se níveis diferentes de atividades de formigas escolhendo-se uma espécie que hospedava membracídeos, o feijão guandu, e outra que não hospedava, o café. Várias espécies de membracídeos são conhecidas por atrair formigas devido a oferta natural de açucares proporcionada na forma de "honeydew" expelido por esses insetos (Del-Claro \& Oliveira 1999). Além disso, o nível de atividade de formigas sobre as plantas foi aumentado artificialmente através do uso de solução de açúcar, pois várias das espécies que forrageiam sobre plantas são atraídas por secreções líquidas açucaradas (OliveIRA \& BRANDÃo 1991; DELClaro \& Oliveira 1999; Oliveira \& Freitas 2004; Moreira \& DelClaro 2005). Como, por exemplo, as espécies de Camponotus e Brachymyrmex que foram os gêneros de formigas mais comuns sobre as plantas durante a realização dos experimentos.

Durante os experimentos, a presença de membracídeos sobre as plantas de feijão guandu representou uma fonte renovável de alimento para as formigas, o que explicaria o maior número de espécies sobre essas plantas, já que formigas foram vistas coletando "honeydew" dos membracídeos encontrados nas plantas de feijão guandu. Os exsudados de hemípteros podem, em algumas situações, ser a principal fonte de alimento para colônias de formigas (Rico-GraY 1989, 1993). Por outro lado, as plantas de café não hospedavam qualquer hemíptero que pudesse oferecer alimentos para as colônias no ambiente, mas a presença de formigas sobre as plantas pode estar associada à oferta de locais para nidificação, representada pela disponibilidade de galhos das próprias plantas que secam e permanecem durante algum tempo ligados a elas (LARSEn \& PHILPOTT 2010). Isto pode explicar a maior ocorrência das espécies $C$. crassus, Crematogaster sp. e Monomorium sp. nas plantas experimentais (LongINo 2005).

Em plantas de feijão guandu a atividade de formigas já era maior pela presença dos membracídeos, mas a aplicação da solução de açúcar incrementou ainda mais essa atividade. Já nas plantas de café borrifadas com solução de açúcar, o aumento na atividade de formigas pode ser explicado pela resposta de formigas que habitassem os galhos secos da planta borrifada ou menos provavelmente de formigas do solo que subiram na planta. Há evidências de que a presença de fontes de açúcar no solo estimula as formigas a subirem em plantas das proximidades (DEL-CLARO
\& Oliveira 1996).

Neste estudo usamos a abordagem experimental para avaliar o potencial de predação das formigas em plantas cultivadas usando modelos artificiais de ovos de insetos. Para testar a importância do nível de atividade de formigas, foram usadas plantas com e sem membracídeos, que oferecem uma fonte natural de açucares para formigas (DEL-Claro \& Olvivira 1999). O nível de atividade das formigas foi também alterado artificialmente através do uso de solução de açúcar borrifada sobre a folhagem das plantas. Os resultados apresentados comprovaram o relevante papel ecológico das formigas. Esses organismos podem atuar como agentes de controle biológico de pragas das culturas.

\section{AGRADECIMENTOS}

Aos dois revisores anônimos pelas sugestões em uma versão prévia do manuscrito. À FAPERJ, pela bolsa de Iniciação Cientifica a L.X.T. Chevalier (Proc. 151.902/2006) e DSG (Proc. 150.902/2007); e ao CNPq pela bolsa concedida a A.J. MayhéNunes. À EMBRAPA-Agrobiologia pelas facilidades criadas para o trabalho.

\section{REFERÊNCIAS}

Andrade, I., 1997. Avaliação experimental de variações espaciais e temporais no ataque de larvas de Lepidoptera. Dissertação (Mestrado em Ecologia) - Universidade Estadual deCampinas. 8op.

Bentley, B.L., 1977. Extrafloral nectaries and protection by pugnacious bodyguards. Annual Review of Ecology And Systematics, 8: 407-427.

Bolton, B., 1994. Identification guide to the ant genera of the world. Cambridge, MA: Harvard University Press.

Buckley, R., 1987. Interactions involving plants, Homoptera, and ants. Annual Review of Ecology and Systematics, 18: 111138.

Costa, F.M.C.B., A.T. Oliveira-Filho \& P.S. Oliveira, 1992. The role of extrafloral nectaries in Qualea grandiflora (Vochysiaceae) in limiting herbivory: an experiment of ant protection in cerrado vegetation. Ecological Entomology, 17: 362-365.

Del-Claro, K. \& P.S. Oliveira, 1996. Honeydew flicking by treehoppers provides cues to potential tending ants. Animal Behaviour, 51: 1071-1075.

Del-Claro, K. \& P.S. Oliveira, 1999. Ant-homoptera interactions in a Neotropical Savanna: the honeydew-producing treehopper Guayaquila xiphias (Membracidae) and its associated ant fauna on Didymopanax vinosum (Araliaceae). Biotropica, 31: $135-144$.

Dutra, H.P., A.V.L. Freitas \& P.S. Oliveira, 2006. Dual attraction in the Neotropical shrub Urera baccifera (Urticaceae): The role of ant visitation to pearl bodies and fruits in herbivore deterrence and leaf longevity. Functional Ecology, 20: 252260.

Galetti, M., C.P. Alves-Costa \& E. Cazetta, 2003. Effects of forest fragmentation, anthropogenic edges and fruit colour on the consumption of ornithocoric fruits. Biological Conservation, 111: 269-273.

Hölldobler, B. \& E.O. Wilson, 1990. The Ants. Cambridge, Belknap Press of Harvard University Press, 630p.

Ibarra-Núnez, G.J.A. Garcia, J.A.Lopez\&J.P.Lachaud, 2001. Prey Analysis in the Diet of Some Ponerine Ants (Hymenoptera: Formicidae) and Web-building Spiders (Araneae) in Coffee Plantations in Chiapas, Mexico. Sociobiology, 37: 723-756.

Junqueira, L.K., E. Diehl \& E. Diehl-Fleigh, 2001. Formigas (Hymenoptera: Formicidae) visitantes de Ilexparaguariensis (Aquafoliaceae). Neotropical Entomology, 30: 161-164.

Koptur, S., 1984. Experimental evidence for defense of Inga (Mimosoideae) samplings by ants. Ecology, 65: 1787-1793.

Lapolla, J.S.; Brady, S.G. \& S.O. Shattuck, (2010). Philogeny and taxonomy of Prenolepis genus-group of ants (Hymenoptera: Formicidae). Systematic Entomology, 35: 118-131. 
Larsen, A. \& Philpott, S.M., 2010. Twig-Nesting Ants: The Hidden Predators of the Coffee Berry Borer in Chiapas, Mexico. Biotropica, 42: $342-347$.

Longino, J.T., 2005. Ants of Costa Rica. Disponível em: <www.evergreen. edu/ants/antsofcostarica.html> [Acesso: 20 out 2012].

Machado, G. \& A.V.L. Freitas, 2001. Larval defense against ant predation in the butterfly Smyrna blomfildia. Ecological Entomology, 26: 436-439.

Moreira, V.S.S. \& K. Del-Claro, 2005. The outcomes of an anttreehopper association on Solanum lycocarpum St. Hill: increased membracid fecundity and reduced damage by chewing herbivores. Neotropical Entomology, 34: 881-887.

Oliveira, P.S. \& C.R.F. Brandão, 1991. The ant community associated with extrafloral nectaries in the Brazilian cerrados, p. 198-212. In: Huxley, C.R. \& D.F. Cutler (eds) Ant-plant interactions. Oxford, Oxford University Press, p. 624.

Oliveira, P.S. \& A.V.L. Freitas, 2004. Ant-plant-herbivore interactions in the Neotropical cerrado savanna. Naturwissenschaften, 91: 557-570.

Oliveira, P.S., A.T. Oliveira- Filho \& R. Cintra, 1987. Ant foraging on ant-inhabited Triplaris (Polygonaceae) in western Brazil: a field experiment using live termite-baits. Journal of Tropical Ecology, 3: 193-200.

Philpott, S.M. \& I. Armbrecht, 2006. Biodiversity in tropical agroforests and the ecological role of ants and ant diversity in predatory function. Ecological Entomology, 31: 369-377.

Philpott, S.M., I. Perfecto \& J. Vandermeer, 2006. Effects of management intensity and season on arboreal ant diversity and abundance in coffee agroecosystems. Biodiversity and Conservation, 15: 139-155.
Queiroz, J.M. \& P.S. Oliveira, 2001. Tending ants protect honeydew producing whiteflies. Environmental Entomology, 30: 295-297.

Queiroz, J.M., F.S. Almeida \& M.P.S. Pereira, 2006. Conservação da biodivesidade e o papel das formigas (Hymenoptera: Formicidae) em agroecossistemas. Floresta \& Ambiente, 13: 37-45.

Rashbrook, V.K., S.G. Compton \& J.H. Lawton 1992. Antherbivore interactions: reasons for the absence of benefits to a fern with foliar nectaries. Ecology, 73: 2167-2174.

Resende, A.L.S., E.E. Silva, V.B. Silva, R.L.D. Ribeiro, J.G.M. Guerra \& E.L. Aguiar-Menezes, 2006. Primeiro registro de Lipaphis pseudobrassicae Davis (Hemiptera: Aphididae) e sua associação com insetos predadores, parasitóides e formigas em couve (Cruciferae) no Brasil. Neotropical Entomology, 35: 551-555

Rico-Gray, V., 1989. The importance of floral and circum-floral nectar to ants inhabiting dry tropical lowlands. Biological Journal of Linnean Society, 38: 173-181.

Rico-Gray, V., 1993. Use of plant-derived food resources by ants in the dry tropical lowlands of coastal Veracruz, Mexico. Biotropica, 25: 301-315.

Vandermeer, J., I. Perfecto, G.I. Nuñez, S.M. Philpott \& A.G. Ballinas, 2002. Ants (Azteca sp.) as potencial biological control agents in shade coffee production in Chiapas, México. Agroforestry Systems, 56: 271-276.

Recebido em: 27/10/2012

Aceito em: 07/02/2013

\section{Como citar este artigo:}

L.X.T. Chevalier, D.S. Gomes, A.J. Mayhé-Nunes \& J.M. Queiroz, 2013. Potencial de Formigas (Hymenoptera: Formicidae) como Agentes Antiherbívoros em Cultivo de Café (Coffea canephora Pierre) e Feijão Guandu [Cajanus cajans (L.) Millsp]. EntomoBrasilis, 6(2): 113-118. Acessível em: http://www.periodico.ebras.bio.br/ojs/index.php/ebras/article/view/291. doi:10.12741/ebrasilis.v6i2.291 